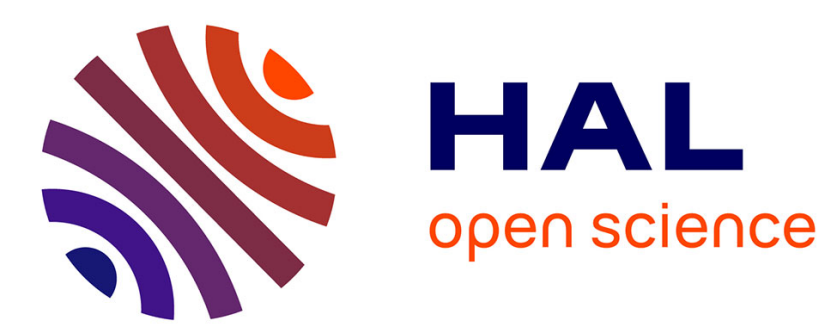

\title{
Fast input-free observers for LPV discrete-time systems
} Mirko Fiacchini, Gilles Millérioux

\section{To cite this version:}

Mirko Fiacchini, Gilles Millérioux. Fast input-free observers for LPV discrete-time systems. 51st IEEE Conference on Decision and Control, CDC 2012, Dec 2012, Maui, Hawaii, United States. pp.CDROM. hal-00735070

\section{HAL Id: hal-00735070 \\ https://hal.science/hal-00735070}

Submitted on 25 Sep 2012

HAL is a multi-disciplinary open access archive for the deposit and dissemination of scientific research documents, whether they are published or not. The documents may come from teaching and research institutions in France or abroad, or from public or private research centers.
L'archive ouverte pluridisciplinaire HAL, est destinée au dépôt et à la diffusion de documents scientifiques de niveau recherche, publiés ou non, émanant des établissements d'enseignement et de recherche français ou étrangers, des laboratoires publics ou privés. 


\title{
Fast input-free observers for LPV discrete-time systems
}

\author{
Mirko Fiacchini $^{1,2}$ and Gilles Millérioux ${ }^{1,2}$
}

\begin{abstract}
This paper deals with fast input-free partial state estimation for discrete-time LPV systems through functional observers. The system inputs are assumed to be unknown and then the observers reduce to functions of finite sequences of the output only. The existence of the observer is proved by resorting to both the notion of inverse system and the concept of maximal A-invariant subspaces. A constructive approach to derive the explicit equations of the observer is provided. An example illustrates the efficiency and the computational aspects of the method.
\end{abstract}

\section{INTRODUCTION}

Reconstructing the state vector of a system from its accessible outputs is an important issue in automatic control, in particular for state feedback control law synthesis or diagnosis. In order to estimate the state of a system, it may be assumed that all system inputs are measurable. On the other hand, for systems with some unmeasurable inputs, specific methods should be used. A first solution is to assume that the unmeasurable quantities satisfy a given dynamics. That amounts to modeling the unknown inputs as the response of a suitable a priori chosen dynamical system. An augmented system is then constructed and an observer estimates the augmented state. On the other hand, we may face the situation when the inputs are completely unknown and not measurable. In such a case, we can resort to an unknown input, also called input-free, state reconstruction. The issue of designing such observers goes back to the 70's with a pioneering work [11].

In this paper, we are interested in functional nonasymptotic input-free observers. Functional observers are observers which aim at reconstructing a particular (often linear) function of the state (possibly of the input as well). Functional observers for linear systems have been widely addressed since the pioneering work [14]. A complete framework providing necessary and sufficient conditions of stability and design procedure has been reported in [8]. The books [7] and more recently [20] present a state of the art of research in this field. The consideration of nonlinear discretetime systems with unknown inputs is rather scarce and motivate the present work. The concept of non-asymptotic reconstruction (with known or unknown inputs) has been mainly investigated through the algebraic framework. In short, algebraic observability means that, each state variable can be expressed as an algebraic function of the output, the

This work was partially supported by the CPER MISN Région Lorraine (AOC Project 2011), Fédération Charles Hermite, ESSTIN and INRIA.

1 Université de Lorraine, CRAN, UMR 7039, ESSTIN, 2 rue Jean Lamour, Vandœuvre-lès-Nancy Cedex, 54506, France

2 CNRS, CRAN, UMR 7039, France input (if known) and a finite number of shifts in the discretetime case [18] [10]. Let us notice that different terms are used in the literature to define the same concept: non-asymptotic, dead-beat, finite-time, fast.

The approach proposed in this paper for the design of dead-beat functional observer is based on results of settheory for control, in particular the concept of invariance. Invariance of a set or a subspace of the state space is related to many classical topic in control, such as stability, Lyapunov theory, constrained control. Invariance and settheoretic methods in control appeared at the end of the sixties, see the pioneering work [5], and they raised an increasing interest in the last decades, see in particular the recent monograph [6] and references therein for an exhaustive overview on the topic. The characterization of invariant subspaces, strongly related also with the properties of controllability and observability, has been treated in [2][4]. More recently, invariant subspaces have been used in the context of LPV systems in [1], and for unknow input state estimation for a particular class of nonlinear continuous-time systems, see [12].

This paper deals with discrete-time LPV systems with unknown inputs. The objective is two-fold. First, we want to derive existence conditions under which a given state variable (or a linear combination of the state variables) can be expressed as a function of a finite number of shifted outputs. This in accordance with algebraic observability. Secondly, we aim at providing an explicit solution to the problem, that is deriving the equations of the state reconstructor.

The outline of the paper is the following. Section II is devoted to the problem statement, namely the issue of fast input-free reconstruction. In Section III, the existence of the reconstructor is proved and its equations are derived. The the notions of inverse system and invariant subspaces are used. Two approaches are presented. Computational issues are addressed in Section IV. Finally, an example illustrates the efficiency and the computational aspects of the method. Notation: $\mathbb{N}$ is the set of natural numbers. Given $n \in \mathbb{N}$ denote $\mathbb{N}_{n}=\{x \in \mathbb{N}: 1 \leq x \leq n\}$ and $\overline{\mathbb{N}}_{n}=\mathbb{N}_{n} \cup\{0\}$. We denote with $x^{(i)}$ the $i$-th entry of the vector $x$. Notation $P_{i}$ denotes the $i$-th element of a set of matrices and $x_{k}$ is the realization of the time-dependent vector $x$ at time $k$. We assume the convention that $\prod_{i=0}^{d} P_{i}=P_{d} \ldots P_{0}$. For a matrix $X, X^{\dagger}$ stands for the classical Moore-Penrose generalized inverse of $X$. 


\section{Problem Statement}

Consider discrete-time LPV systems obeying

$$
\left\{\begin{array}{l}
x_{k+1}=A\left(\theta_{k}\right) x_{k}+B\left(\theta_{k}\right) u_{k} \\
y_{k}=C\left(\theta_{k}\right) x_{k}+D\left(\theta_{k}\right) u_{k}
\end{array}\right.
$$

where $x_{k} \in \mathbb{R}^{n}$ is the state vector, $u_{k} \in \mathbb{R}^{m}$ is the control input, $y_{k} \in \mathbb{R}^{p}$ is the output vector. The matrices $A \in \mathbb{R}^{n \times n}$, $B \in \mathbb{R}^{n \times m}, C \in \mathbb{R}^{p \times n}$ and $D \in \mathbb{R}^{p \times m}$ depend on the parameter vector $\theta_{k} \in \mathbb{R}^{t}$ belonging to a known set $\Omega_{\theta}$, possibly unbounded. We are interested in the following issue.

Problem 1: Given $G \in \mathbb{R}^{q \times n}$, provide a condition for the existence of a function $f_{\theta}$ such that

$$
G x_{k}=f_{\theta}\left(y_{k+t_{1}}, \ldots, y_{k+t_{2}}\right),
$$

for every $k \geq 0$, every $x_{k} \in \mathbb{R}^{n}$ and every possible realization of the parameter $\theta_{k} \in \Omega_{\theta}$, with appropriate integers $t_{1}, t_{2}$.

It is noteworthy to point out that this problem is rather general. Indeed, if $G$ is the matrix which selects a part of the components of the state vector, the problem boils down to an analysis issue, that is checking whether the components of $x_{k}$ are algebraically observable. If $G$ is the identity matrix, the problem amounts to check whether the system is algebraically observable. Furthermore, any explicit solution to Problem 1 provides $f_{\theta}$ and $t_{1}, t_{2}$, besides guaranteeing their existence. Then a fast input-free observer is obtained for reconstructing either a part or the full state or a linear combination of its elements, depending on the structure of $G$.

\section{A. Inverse system}

The proposed approach to solve Problem 1 rests on the notion of inverse system of (1) whose definition and structure are borrowed from [19]. Define

$$
\mathscr{O}\left(\theta_{k: k+i}\right)=\left[\begin{array}{c}
C\left(\theta_{k}\right) \\
C\left(\theta_{k+1}\right) A\left(\theta_{k}\right) \\
\vdots \\
C\left(\theta_{k+i}\right) \prod_{l=0}^{i-1} A\left(\theta_{k+l}\right)
\end{array}\right]
$$

the vectors

$$
\begin{aligned}
y_{k: k+i} & =\left[y_{k}^{T}, y_{k+1}^{T}, \ldots, y_{k+i}^{T}\right]^{T}, \\
\theta_{k: k+i} & =\left[\theta_{k}^{T}, \theta_{k+1}^{T}, \ldots, \theta_{k+i}^{T}\right]^{T},
\end{aligned}
$$

and the matrix $I_{m \times r}=\left[\begin{array}{ll}\mathbf{I}_{m} & \mathbf{0}_{m \times(m \cdot r)}\end{array}\right]$. Define also $M\left(\theta_{k: k+i}\right)$ in the following recursive way

$$
M\left(\theta_{k: k+i}\right)=\left[\begin{array}{cc}
D\left(\theta_{k}\right) & \mathbf{0}_{p \times m \cdot(i-1)} \\
\mathscr{O}\left(\theta_{k: k+i}\right) B\left(\theta_{k}\right) & M\left(\theta_{k+1: k+i}\right)
\end{array}\right],
$$

with $M\left(\theta_{k: k}\right)=D\left(\theta_{k}\right)$.

Definition 1: An inverse system of (1) is a system for which, when driven by a sequence of output $y_{k}$ of (1), the trajectory of its state vector $\hat{x}_{k}$ coincides with the trajectory $x_{k}$ of (1) (possibly up to a delay $r$ referred to as inherent delay) whenever both state vectors share the same initialization.
A straightforward extension of the inverse system, given in [15], [19] for switched linear ones, obeys for LPV systems the following state space description

$$
\hat{x}_{k+r+1}=P_{\theta}\left(\theta_{k: k+r}\right) \hat{x}_{k+r}+Q_{\theta}\left(\theta_{k: k+r}\right) y_{k: k+r},
$$

with

$$
\begin{aligned}
& P_{\theta}\left(\theta_{k: k+r}\right)=A\left(\theta_{k}\right)-Q_{\theta}\left(\theta_{k: k+r}\right) \mathscr{O}\left(\theta_{k: k+r}\right), \\
& Q_{\theta}\left(\theta_{k: k+r}\right)=B\left(\theta_{k}\right) I_{m \times r} M^{\dagger}\left(\theta_{k: k+r}\right) .
\end{aligned}
$$

Following the same lines as in [15] [19], defining $e_{k}=$ $x_{k}-\hat{x}_{k+r}$ as the state error reconstruction, it can be shown, from (1) and (4) that

$$
e_{k+1}=P_{\theta}\left(\theta_{k: k+r}\right) e_{k}
$$

under the conditions of invertibility, [19]. Hence, if $e_{0}=0$, that is $\hat{x}_{r}=x_{0}$, then $e_{k}=0$ and so $\hat{x}_{k+r}=x_{k}$ for any $k>$ 0 , in accordance with Definition 1. The matrix $P_{\theta}\left(\theta_{k: k+r}\right)$ corresponds to the inverse dynamics. Hence, Problem 1 can be reformulated as follows.

Problem 2: Given $G \in \mathbb{R}^{q \times n}$, provide a condition for the existence of a function $f_{\theta}$ such that

$$
G \hat{x}_{k}=f_{\theta}\left(y_{k+t_{1}^{\prime}}, \ldots, y_{k+t_{2}^{\prime}}\right),
$$

for every $k \geq 0$, every $\hat{x}_{k} \in \mathbb{R}^{n}$ and every possible realization of the parameter $\theta_{k} \in \Omega_{\theta}$, with appropriate integers $t_{1}^{\prime}, t_{2}^{\prime}$.

\section{DESCRIPTION OF THE APPROACH}

First, notice that the parametric dependence of the matrices in (4) and (5) with respect to $\theta_{k: k+r} \in \Omega_{\theta}^{r+1}$ is not linear in general. However, given the set $\Omega_{\theta}$, there always exists a function $p$ such that the system (4) depends linearly on the parameter $\rho_{k}$ defined as $\rho_{k}=p\left(\theta_{k: k+r}\right)$. A set $\Omega_{\rho} \subseteq \mathbb{R}^{d}$ such that $\rho_{k} \in \Omega_{\rho}$ if $\theta_{k: k+r} \in \Omega_{\theta}^{r+1}$ can always be determined as well. To simplify the notation, we define

$$
P\left(\rho_{k}\right)=P_{\theta}\left(\theta_{k: k+r}\right), \quad Q\left(\rho_{k}\right)=Q_{\theta}\left(\theta_{k: k+r}\right), \quad \bar{y}_{k}=y_{k: k+r} .
$$

Following the considerations above, the assumption below can be posed.

Assumption 1: Suppose that $P\left(\rho_{k}\right)$ depends on the parameter $\rho_{k} \in \Omega_{\rho}$ as

$$
P\left(\rho_{k}\right)=\sum_{i=0}^{d} P_{i} \rho_{k}^{(i)} .
$$

Hereafter, unless otherwise stated, Assumption 1 will hold.

Remark 1: Notice that no assumptions are done on $\Omega_{\theta}$, then nor on $\Omega_{\rho}$. In fact $\Omega_{\rho}$ can be whatever subset in the space of the parameter $\rho$. Furthermore, the approach could be easily generalized to more general parameter dependencies, polynomial ones for instance.

A sufficient condition for Problem 2 to have a solution follows.

Proposition 1: There is a solution to Problem 2 with $t_{1}^{\prime}=$ $-K-r-1$ and $t_{2}^{\prime}=-1$ if $K$ and $P_{i}$, for $i \in \overline{\mathbb{N}}_{d}$, are such that

$$
G \prod_{k=0}^{K} P_{i_{k}}=0,
$$

for all $\mathscr{I}=\left[i_{0}, \ldots, i_{K}\right]^{T} \in \overline{\mathbb{N}}_{d}^{K+1}$. 
Proof: By definition we have

$$
\begin{aligned}
& G \hat{x}_{k+r+1}=G \sum_{i_{0} \in \overline{\mathbb{N}}_{d}} \rho_{k}^{\left(i_{0}\right)} P_{i_{0}} \hat{x}_{k+r}+G Q\left(\rho_{k}\right) \bar{y}_{k}, \\
& G \hat{x}_{k+r+2}=G \sum_{\mathscr{I} \in \overline{\mathbb{N}}_{d}^{2}} \prod_{l=0}^{1} \rho_{k+l}^{\left(i_{l}\right)} P_{i_{l}} \hat{x}_{k+r}+G Q\left(\rho_{k+1}\right) \bar{y}_{k+1}+ \\
& \quad+G \sum_{\mathscr{I} \in \mathbb{N}_{d}^{1}} \rho_{k}^{\left(i_{0}\right)} P_{i_{0}} Q\left(\rho_{k}\right) \bar{y}_{k}, \\
& \quad \ldots \quad \\
& G \hat{x}_{k+r+K+1}=G \sum_{\mathscr{I} \in \overline{\mathbb{N}}_{d}^{K+1} \prod_{l=0}^{K} \rho_{k+l}^{\left(i_{l}\right)} P_{i_{l}} \hat{x}_{k+r}+G Q\left(\rho_{k+K}\right) \bar{y}_{k+K}+}^{K} \quad+G \sum_{j=0}^{K-1} \sum_{\mathscr{I} \in \overline{\mathbb{N}}_{d}^{j+1}} \prod_{l=K-j}^{\rho_{k+l}^{\left(i_{l}\right)} P_{i_{l}}} Q\left(\rho_{k+K-1-j}\right) \bar{y}_{k+K-1-j},
\end{aligned}
$$

where $\mathscr{I}=\left[i_{0}, \ldots, i_{k}\right]^{T} \in \overline{\mathbb{N}}_{d}^{k+1}$ for all $k \in \overline{\mathbb{N}}_{K}$. If for every $\mathscr{I} \in \overline{\mathbb{N}}_{d}^{K+1}$ condition (8) holds, then

$$
\begin{aligned}
& G x_{k+K+1}=G \hat{x}_{k+r+K+1}=G Q\left(\rho_{k+K}\right) \bar{y}_{k+K}+ \\
& \quad+G \sum_{j=0}^{K-1} \sum_{\mathscr{I} \in \overline{\mathbb{N}}_{d}^{j+1}} \prod_{l=K-j}^{K} \rho_{k+l}^{\left(i_{l}\right)} P_{i_{l}} Q\left(\rho_{k+K-1-j}\right) \bar{y}_{k+K-1-j} .
\end{aligned}
$$

Finally, after a suitable shift, we get that (9) completes the proof and shows that $f_{\theta}$ involves the output $y_{k+l}$ (and the parameter $\theta_{k+l}$ ) for $-K-r-1 \leq l \leq-1$.

Remark 2: Some sequence among all the admissible ones are not allowed since, by definition, $P\left(\rho_{i+1}\right)$ is not independent with respect to $P\left(\rho_{i}\right)$. Such sequences could be discarded from the analysis.

In the following, two computation-oriented conditions for (8) to hold are presented. They involve the concept of invariant subspaces. First we introduce the concept of Ainvariant subspace, see the monograph [4] for details.

Definition 2 (A-invariant subspace): Given a linear transformation $A: \mathbb{R}^{n} \rightarrow \mathbb{R}^{n}$ an A-invariant is a subspace $\mathscr{V} \subseteq \mathbb{R}^{n}$ such that

$$
A \mathscr{V} \subseteq \mathscr{V}
$$

Given the parameter-dependent family of linear transformations $A(\rho): \mathbb{R}^{n} \rightarrow \mathbb{R}^{n}$, for $\rho \in \Omega_{\rho}$, a subspace $\mathscr{V} \subseteq \mathbb{R}^{n}$ is a robust A-invariant if

$$
A(\rho) \mathscr{V} \subseteq \mathscr{V}, \quad \forall \rho \in \Omega_{\rho}
$$

Geometrically, $\mathscr{V}$ is an A-invariant subspace if every trajectory starting inside it, remains confined within it.

Proposition 2: Given a linear transformation $A: \mathbb{R}^{n} \rightarrow \mathbb{R}^{n}$, its $\operatorname{kernel} \operatorname{ker}(A)$ is an A-invariant.

Proof: Denote with $T$ a basis matrix of $\operatorname{ker}(A)$. By definition (see [4]), $\operatorname{ker}(A)$ is A-invariant for the linear transformation $A: \mathbb{R}^{n} \rightarrow \mathbb{R}^{n}$ if and only if there exists a matrix $X$ such that

$$
A T=T X .
$$

Since $T$ is a basis of $\operatorname{ker}(A)$ it follows that $A T=0$, and then (10) holds merely posing $X=0$.

Definition 3: Given $P\left(\rho_{k}\right)$ as in Assumption 1, define

$$
\mathscr{P}=\bigcap_{i=0}^{d} \operatorname{ker}\left(P_{i}^{T}\right) \text {. }
$$

Moreover, denote with $M \in \mathbb{R}^{n \times g}$ a basis matrix of $\mathscr{P}$ and $N \in \mathbb{R}^{n \times(n-g)}$ a basis of its complement.

Notice that the definition of $\mathscr{P}$ above involves the transposes of the matrices $P_{i}$ for all $i \in \overline{\mathbb{N}}_{d}$.

Proposition 3: The set $\mathscr{P}$ as in Definition 3 is A-invariant for the parameter-dependent system given by

$$
z_{k+1}=P_{T}\left(\rho_{k}\right) z_{k} \quad \text { with } \quad P_{T}\left(\rho_{k}\right)=\sum_{i=0}^{d} \rho_{k}^{(i)} P_{i}^{T}=P\left(\rho_{k}\right)^{T} .
$$

Proof: From Proposition 2, $\mathscr{P}$ is an A-invariant for every $P_{i}^{T}$ with $i \in \overline{\mathbb{N}}_{d}$ and $M$ a basis matrix of $\mathscr{P}$. By definition, $\mathscr{P}$ is A-invariant also for the system whose dynamics is given by (11) if and only if there exists $X$ such that

$$
0=\sum_{i=0}^{d} \rho_{k}^{(i)} P_{i}^{T} M=M X
$$

that holds posing $X=0$.

\section{A. Kernel-based approach}

A sufficient condition for Proposition 1 to hold, and hence an admissible solution to Problem 2, follows.

Proposition 4: Consider the system (4), the set $\mathscr{P}$ and the matrix $M$ as in Definition 3. If $G \in \mathbb{R}^{q \times n}$ is such that the columns of $G^{T}$ are in $\mathscr{P}$, i.e. there is $X \in \mathbb{R}^{d \times g}$ such that

$$
G=X M^{T}
$$

then there exists a solution of Problem 2 with $K=1$.

Proof: The result stems directly from definition of $\mathscr{P}$, as if the rows of $G$ are in $\mathscr{P}$ then

$$
G P_{i}=0, \quad \forall i \in \overline{\mathbb{N}}_{d},
$$

and then the condition (8), sufficient for solving the Problem 2, holds with $K=1$.

The result in Proposition 4 is implicitly based on the fact that the set $\mathscr{P}$ is an A-invariant for the system (4) as proved in Proposition 3. On the other hand, this condition requires that the dynamics of the system (4) would reach the particular A-invariant subspace in one step. This is obviously restrictive but provides an interesting insight on the problem and a clue on its possible alternative as shown below.

\section{B. A-invariant-based approach}

An alternative to Proposition 4 for condition (8) to hold is now provided.

Definition 4: Let Assumption 1 hold for the system (4). Denote with $\mathscr{G}$ the maximal A-invariant subspace for $P_{i}$, for $i \in \overline{\mathbb{N}}_{d}$, contained in $\operatorname{ker}(G)$, with $g=\operatorname{dim}(\mathscr{G}), \bar{T} \in \mathbb{R}^{n \times g}$ its basis matrix and $\hat{T} \in \mathbb{R}^{n \times h}$ a matrix such that $T=[\bar{T}, \hat{T}] \in$ $\mathbb{R}^{n \times n}$ is nonsingular.

An interesting property, based on the results presented in [4], is the fact that the dynamics of a system on every Ainvariant subspace can be decoupled by the dynamics on complementary subspace. 
Proposition 5: Let Assumption 1 hold for the system (4) and consider $\mathscr{G}$ and $T$ as in Definition 4 . Then $\mathscr{G}$ is Ainvariant for $P\left(\rho_{k}\right)$ and there exist $\bar{P}\left(\rho_{k}\right) \in \mathbb{R}^{g \times g}, \tilde{P}\left(\rho_{k}\right) \in$ $\mathbb{R}^{g \times h}$ and $\hat{P}\left(\rho_{k}\right) \in \mathbb{R}^{h \times h}$, linear in $\rho_{k}$ such that:

$$
P\left(\rho_{k}\right)=T\left[\begin{array}{cc}
\bar{P}\left(\rho_{k}\right) & \tilde{P}\left(\rho_{k}\right) \\
0 & \hat{P}\left(\rho_{k}\right)
\end{array}\right] T^{-1}
$$

for every $\rho_{k} \in \Omega_{\rho}$.

Proof: First notice that, by definition of A-invariant subspaces, if $\mathscr{G}$ is an A-invariant for all $P_{i}$ with $i \in \overline{\mathbb{N}}_{d}$ then it is A-invariant for every single $P_{i}$. This implies that for all $i \in \overline{\mathbb{N}}_{d}$ there exist $\bar{P}_{i} \in \mathbb{R}^{g \times g}, \tilde{P}_{i} \in \mathbb{R}^{g \times h}, \hat{P}_{i} \in \mathbb{R}^{h \times h}$ and $\bar{T} \in \mathbb{R}^{g \times g}, \hat{T} \in \mathbb{R}^{h \times h}$ such that

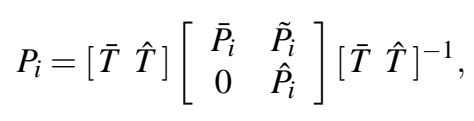

with $\bar{T}$ basis of $\mathscr{G}$, see Theorem 3.2.1 in [4]. Posing

$$
\bar{P}\left(\rho_{k}\right)=\sum_{i=0}^{d} \bar{P}_{i} \rho_{k}^{(i)}, \quad \tilde{P}\left(\rho_{k}\right)=\sum_{i=0}^{d} \tilde{P}_{i} \rho_{k}^{(i)}, \quad \hat{P}\left(\rho_{k}\right)=\sum_{i=0}^{d} \hat{P}_{i} \rho_{k}^{(i)}
$$

then (12) holds and $\mathscr{G}$ is a robust A-invariant for $P\left(\rho_{k}\right)$ according to (10).

We must further consider the concept of nilpotent semigroups to derive the result which finally solves Problem 2. Let us first recall some basics related to nilpotent semigroups.

Definition 5 (Semigroup): A semigroup $\mathscr{S}$ is a set together with an associative internal law.

It is said to be finite if it has a finite number of elements. If $\mathscr{S}$ is a set of matrices, the associative internal law is the matrix multiplication. We denote by 0 the absorbing element of a semigroup when it exists.

Definition 6 (Nilpotent semigroup): A semigroup $\mathscr{S}$ with an absorbing element 0 is said to be nilpotent if there is a positive integer $t$ such that the internal law applied to any $t$ elements of $\mathscr{S}$ is always equal to 0 . The smallest integer $t$ is called the nilpotency class of $\mathscr{S}$.

If $\mathscr{S}$ is a set of matrices, applying the internal low to any $t$ elements of $\mathscr{S}$ amounts to performing the product of $t$ matrices of $\mathscr{S}$. The absorbing element is in this case the null matrix.

Let us notice that the property of nilpotency for a semigroup is less conservative than a closely related property often encountered in control (see [13]), namely, solvability of a Lie algebra.

Theorem 1: Let Assumption 1 hold for the system (4) and consider $\mathscr{G}$ and $T$ as in Definition 4. If $\hat{P}_{i}$ for all $i \in \overline{\mathbb{N}}_{d}$ generate a nilpotent semigroup of class $t$ then, Problem 2 is solved with $K=t$.

Proof: By construction, $\bar{T}$ is a basis of the A-invariant subspace $\mathscr{G}$ that is contained in $\operatorname{ker}(G)$ then $G \bar{T}=0$. Hence, for all $\mathscr{I}=\left[i_{0}, \ldots, i_{K}\right]^{T} \in \overline{\mathbb{N}}_{d}^{K}$, we have

$$
\begin{aligned}
& G \prod_{k=0}^{K} P_{i_{k}}=G \prod_{k=0}^{K} T\left[\begin{array}{cc}
\bar{P}_{i_{k}} & \tilde{P}_{i_{k}} \\
0 & \hat{P}_{i_{k}}
\end{array}\right] T^{-1}= \\
& =G[\bar{T}, \hat{T}]\left(\prod_{k=0}^{K}\left[\begin{array}{cc}
\bar{P}_{i_{k}} & \tilde{P}_{i_{k}} \\
0 & \hat{P}_{i_{k}}
\end{array}\right]\right) T^{-1}= \\
& =\prod_{k=0}^{K}\left[0_{q \times g}, G \hat{T}\right]\left[\begin{array}{cc}
\bar{P}_{i_{k}} & \tilde{P}_{i_{k}} \\
0 & \hat{P}_{i_{k}}
\end{array}\right] T^{-1}= \\
& =\left[0_{q \times g}, G \hat{T} \prod_{k=0}^{K} \hat{P}_{i_{k}}\right] T^{-1}=0 .
\end{aligned}
$$

The last equality precisely stems from the fact that $\hat{P}_{i}$ with $i \in \overline{\mathbb{N}}_{d}$ generate a nilpotent semigroup of class $K$. Thus condition (8) is satisfied for all $\mathscr{I}=\left[i_{0}, \ldots, i_{K}\right]^{T} \in \overline{\mathbb{N}}_{d}^{K}$, which is sufficient for the existence of a solution to Problem 2. Furthermore, the solution is explicit since the function $f_{\theta}$, that is the input-free reconstructor, obeys (9) in this case.

Remark 3: It can be shown, see [16], that the condition is also necessary for at least two particular cases: when the inherent delay $r$ is equal to zero or if it is equal to one and that $C$ is constant. These two cases encompass a large class of systems.

Remark 4: Clearly, the class of nilpotency $t$, and so $K$ is smaller than or equal to $h$, that is the dimension of the subspace complementary to $\mathscr{G}$ in $\mathbb{R}^{n}$. Then, the bigger the dimension of $\mathscr{G}$, the smaller such a bound, in general. Moreover, notice that for lower values of $q$, number of rows of $G$, the dimension of $\mathscr{G}$ can be higher and then the dimension of matrices $\hat{P}_{i}$ is smaller. This is conceptually reasonable, as the smaller is the partial state to be estimated, the smaller is the subspace within which the system dynamics must converge to zero in finite time.

\section{Computational issues}

In this section, we provide some insights on the computational procedures for obtaining the A-invariant subspaces (Proposition 5) and for determining whether a set of matrices generates a nilpotent semigroup (Theorem 1). According to Definition 2, a subspace $\mathscr{V}$ is A-invariant for a linear system if its image through the linear transformation is contained in $\mathscr{V}$. Well established results on how to generate A-invariant subspaces have been presented in literature, see in particular [4]. We recall here the basic ideas and we provide some practical details on the computation.

First, for a linear system whose dynamics is given by the linear transformation $A: \mathbb{R}^{n} \rightarrow \mathbb{R}^{n}$, two trivial A-invariant subspaces contained in $\mathbb{R}^{n}$ are the origin and the space $\mathbb{R}^{n}$. In general, one can be interested either in the minimal Ainvariant containing a given subspace or the maximal Ainvariant contained in a subspace. The latter case enters our concern. Denoting with $\mathscr{J}$ the maximal A-invariant contained in the subspace $\mathscr{V}, \mathscr{J}$ is computed as illustrated below.

Algorithm 1: Given the subspace $\mathscr{V} \subseteq \mathbb{R}^{n}$ and the linear transformation $A: \mathbb{R}^{n} \rightarrow \mathbb{R}^{n}$ the maximal A-invariant subspace $\mathscr{J}$ is provided by the following iteration:

$$
\begin{aligned}
& \mathscr{J}_{0}=\mathscr{V}, \\
& \mathscr{J}_{i+1}=\mathscr{V} \cap A^{-1} \mathscr{J}_{i}, \quad i=0, \ldots, \bar{n} .
\end{aligned}
$$


The algorithm stops after a finite number of iterations $\bar{n}$, whose upper bound is $n-1$. In particular, the iteration can be stopped as soon as $\mathscr{J}_{i+1}=\mathscr{J}_{i}$.

Remark 5: Notice that, with a slight abuse of notation, the meaning of the symbol $A^{-1}$ does not denote the inverse of matrix $A$, but it is the operator that associates to a subspace its inverse image, that is, given the subspace $\mathscr{J}_{i} \subseteq \mathbb{R}^{n}$ :

$$
A^{-1} \mathscr{J}_{i}=\left\{x \in \mathbb{R}^{n}: A x \in \mathscr{J}_{i}\right\} .
$$

Indeed, it is worth pointing out that the inverse image of a subspace can be computed also for linear transformation given by noninvertible matrices, as in our case. For instance, the inverse image of the origin is given by the kernel of $A$.

Remark 6: It can be proved that the same sequence of subspaces $\mathscr{J}_{i}$ with $i=0, \ldots, \bar{n}$ is generated by the iteration:

$$
\mathscr{J}_{i+1}=\mathscr{J}_{i} \cap A^{-1} \mathscr{J}_{i}, \quad i=0, \ldots, \bar{n} .
$$

Remark 7: The Algorithm 1, either implementing the iteration (14) or (15), is based, substantially, on the computation of the intersection between subspaces and of the inverse image of a subspace. We provide hereafter a sketch of the procedure. Consider two subspaces $\mathscr{V} \in \mathbb{R}^{n}$ and $\mathscr{U} \in \mathbb{R}^{n}$, whose basis matrices are $V \subseteq \mathbb{R}^{n \times v}$ and $U \subseteq \mathbb{R}^{n \times u}$. The subspace $\mathscr{V} \cap A^{-1} \mathscr{U}$ is given by the vectors $x \in \mathbb{R}^{n}$ that can be expressed as a linear combination of the columns of $V$ and their image through $A$ as a linear combination of those of $U$. Then, in practice, $x \in \mathscr{V} \cap A^{-1} \mathscr{U}$ if there exists $y \in \mathbb{R}^{v}$ and $z \in \mathbb{R}^{u}$ such that $x=V y$ and $A x=U z$, that means such that $A V y=U z$. Hence, $\mathscr{V} \cap A^{-1} \mathscr{U}$ can be obtained by computing the kernel of $[A V, U]$.

Interestingly for our purpose, the Algorithm 1 can be extended to the case of LPV systems, see [1], [4]. In general, given a linear transformation $A(\rho)$ depending on the parameter $\rho$, the maximal A-invariant subspace, referred to as robust, containing $\mathscr{V}$ is given by the following algorithm.

Algorithm 2: Given the subspace $\mathscr{V} \subseteq \mathbb{R}^{n}$ and the linear parameter dependent transformation $A(\bar{\rho}): \mathbb{R}^{n} \rightarrow \mathbb{R}^{n}$, with $\rho \in \Omega_{\rho}$ the maximal robust A-invariant subspace $\mathscr{J}$ is provided by the following iteration:

$$
\begin{aligned}
& \mathscr{J}_{0}=\mathscr{V}, \\
& \mathscr{J}_{i+1}=\mathscr{J}_{0} \cap \bigcap_{\rho \in \Omega_{\rho}} A(\rho)^{-1} \mathscr{J}_{i}, \quad i=0, \ldots, \bar{n} .
\end{aligned}
$$

Similarly to the linear case, the algorithm stops after a finite number $\bar{n}$ of iterations, smaller or equal than $n-1$. The problem for computing robust A-invariant subspaces appears when the set $\Omega_{\rho}$ is uncountable. Approximation methods are available. For the case of finite $\Omega_{\rho}$, the Algorithm 2 provides the maximal robust A-invariant contained in $\mathscr{V}$ after a finite number of operations. This is precisely the case for LPV systems considered in Assumption 1, for which the Algorithm 2 delivers the A-invariant after a finite number of iterations.

As for the computational aspects regarding the nilpotency property, we must recall the Levitsky's theorem (Theorem 2.1.7 stated in [17]).

Theorem 2 (Levitsky's theorem): Any nilpotent matrices can be triangularized.
In other words, all the matrices of a same nilpotent semigroup can be triangularized by means of a common change of basis. The triangularization can be performed for example with the algorithm given in [9]. Such an algorithm is quite appealing since it allows the triangularization to be performed with a complexity which is linear with respect to the number of matrices and polynomial with respect to the dimension (see details for flat ouput characterization purposes in [16]).

\section{ILLUSTRATIVE EXAMPLE}

We consider the LPV system (1) whose dynamics is given by the following matrices

$$
A=\left[\begin{array}{ccc}
-4 & 1 & 0 \\
\theta_{k} & 0 & 1 \\
-2 & 0 & 0
\end{array}\right], B=\left[\begin{array}{l}
0 \\
1 \\
1
\end{array}\right], C=\left[\begin{array}{l}
1 \\
0 \\
0
\end{array}\right]^{T}, D=0 .
$$

This system is a SISO one. Hence, since $D=0, C B=0$ and $C A\left(\theta_{k}\right) B \neq 0$ for all $k$, it can be inferred that the inherent delay is $r=2$. Hence, the related inverse systems (4) is characterized (see (5)) by the parameter-dependent matrix

$$
P\left(\theta_{k}\right)=\left[\begin{array}{ccc}
-4 & 1 & 0 \\
-16 & 4 & 0 \\
-14-\theta_{k} & 4 & -1
\end{array}\right]
$$

that is affine in $\theta_{k} \in \mathbb{R}$. Here, the function $p$ such that the matrix $P_{\theta}\left(\theta_{k}\right)$ depends linearly on a parameter $\rho_{k}$ is merely the function $\rho_{k}=\left[1, \theta_{k}\right]^{T}$. The matrices $P_{i}$, as in Assumption 1 , are given by

$$
P_{0}=\left[\begin{array}{ccc}
-4 & 1 & 0 \\
-16 & 4 & 0 \\
-14 & 4 & -1
\end{array}\right], \quad P_{1}=\left[\begin{array}{ccc}
0 & 0 & 0 \\
0 & 0 & 0 \\
-1 & 0 & 0
\end{array}\right]
$$

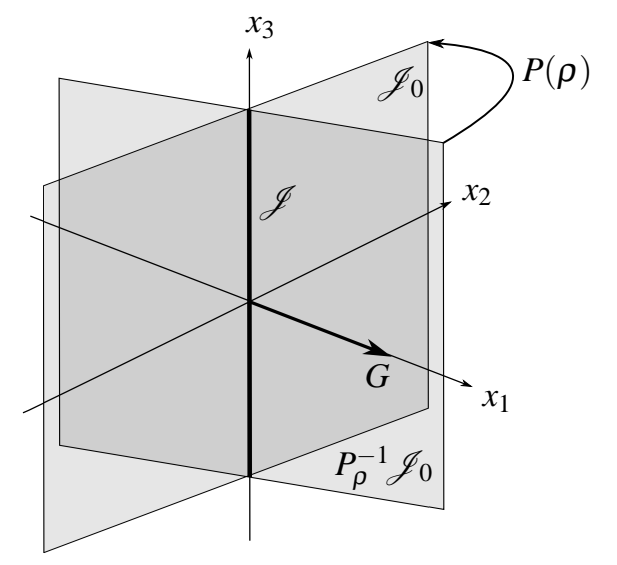

Fig. 1. Sequence of subspaces $\mathscr{J}_{i}$ and $\mathscr{J}$ for $G=[1,0,0]$.

Consider Problem 2 with $G=[1,0,0]$. Then the kernel of $G$ is the plane $x_{k}^{(1)}=0$. A basis of the kernel is given by the vectors $[0,1,0]^{T}$ and $[0,0,1]^{T}$. Let us check whether Theorem 1 is fulfilled. To do so, and as explained in Section IV, we must resort to Algorithm 2. Iteration (15) leads to a sequence of subspaces $J_{i}$, illustrated in Figure 1 , and provides the maximal robust A-invariant subspace 
$\mathscr{J}$ in $\operatorname{ker}(G)$. The basis of $\mathscr{J}$ is $\bar{T}=[0,0,1]^{T}$. After computing the orthogonal basis $\hat{T}$, the affine LPV subsystem related to the orthogonal subspace is computed according to Proposition 5 and is given by matrices

$$
\hat{P}_{0}=\left[\begin{array}{cc}
-4 & 1 \\
-16 & 4
\end{array}\right], \quad \hat{P}_{1}=\left[\begin{array}{ll}
0 & 0 \\
0 & 0
\end{array}\right] .
$$

It turns out that Theorem 1 is fulfilled because $\hat{P}_{0}$ and $\hat{P}_{1}$ are both nilpotent (necessary condition) and generate a nilpotent semigroup with class of nilpotency $t=1$. Hence, $K=1$. We obtain the same result if we consider Problem 2 with $G=[0,1,0]$. Actually, it is worth noticing that the same $\mathscr{J}$ is obtained for every $G$ whose columns lie in the subspace spanned by $[1,0,0]^{T}$ and $[0,1,0]^{T}$. As stressed in the proof of Theorem 1, an explicit solution to Problem 2 can be obtained through (9). Finally, by a direct consequence of the definition of the inverse system, the equality $\hat{x}_{k+r}=x_{k}$ applies and we can thereby directly infer an explicit solution to Problem 1. By considering respectively $G=[1,0,0]$ and $G=[0,1,0]$, the function $f_{\theta}$ of the reconstructor obeys

$$
\begin{aligned}
& x_{k}^{(1)}=y_{k} \\
& x_{k}^{(2)}=y_{k+1}+4 y_{k}
\end{aligned}
$$

The result is obvious for $x_{k}^{(1)}$ that is for $G=[1,0,0]$.

Summarizing, it has been shown that the first two components of $x_{k}$ are algebraically observable and (16) gives an explicit fast input-free observer. Now, considering $G=$ $[0,0,1]$, it turns out that, after applying the Algorithm 2, the resulting maximal robust A-invariant contained in the kernel of $G$, that is $\mathscr{J}$, is the origin, see Figure 2. As it turns out, Theorem 1 is not fulfilled because the resulting matrices $P_{0}$ and $P_{1}$ do not generate a nilpotent semigroup. In fact, this is explained by noticing that $P_{0}$ is not nilpotent, having an eigenvector 1 .

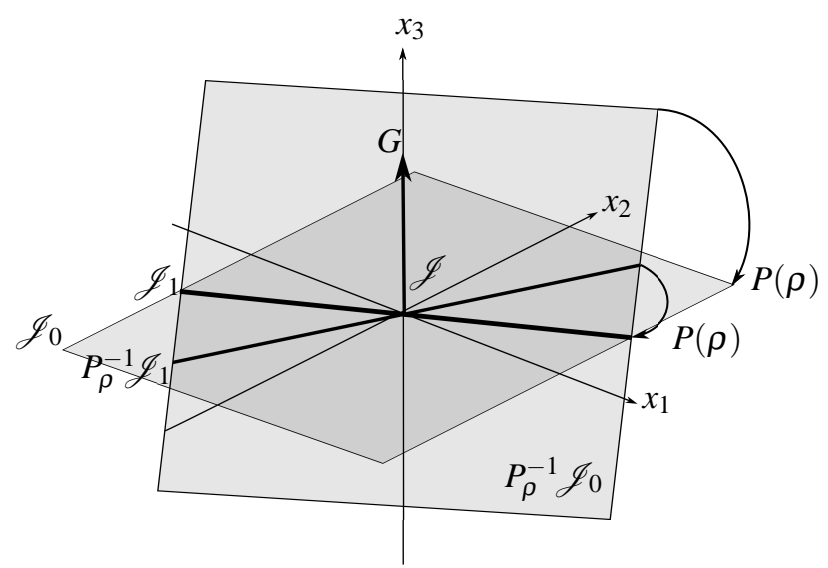

Fig. 2. Sequence of subspaces $\mathscr{J}_{i}$ and $\mathscr{J}$ for $G=[0,0,1]$.

\section{CONCLUSiOnS}

An approach for testing algebraic observability and designing fast input-free state reconstructors for discrete-time LPV systems has been presented. The existence conditions have been derived. They are based on the notion of inverse system, invariant subspaces and nilpotent semigroups. The proof is constructive since it provides a way of deriving the equations of the reconstructor. The results apply for SISO and MIMO systems and without restriction on the relative degree. The results sound interesting to more general cases like LPV systems with polynomial dependence or LDI systems.

\section{REFERENCES}

[1] G. Balas, J. Bokor, and Z. Szabó. Invariant subspaces for lpv systems and their applications. IEEE Transaction on Automatic Control, 48:2065-2069, 2003.

[2] G. Basile and G. Marro. Controlled and conditioned invariant subspaces in linear system theory. Journal of Optimization Theory and Applications, 3:306-315, 1969.

[3] G. Basile and G. Marro. On the robust controlled invariant. Systems and Control Letters, 9:191-195, 1987.

[4] G. Basile and G Marro. Controlled and Conditioned Invariants in Linear system Theory. Prentice Hall, 1992.

[5] D. P. Bertsekas. Infinite-time reachability of state-space regions by using feedback control. IEEE Transactions on Automatic Control, 17:604-613, 1972.

[6] F. Blanchini and S. Miani. Set-Theoretic Methods in Control. Birkhäuser, 2008.

[7] C. T. Chen. Linear System Theory and Design. Oxford Series in Electrical and Computer Engineering, 1984.

[8] M. Darouach. Existence and design of functional observers for linear systems. IEEE Trans. Autom. Control, 45(5):940-943, 2000.

[9] C. Dubi. An algorithmic approach to simultaneous triangularization. Linear Algebra and its Applications, 430(11-12):2975 - 2981, 2009.

[10] R. Germundsson and K. Forsman. A constructive approach to algebraic observability. In Proceedings. 30th IEEE Conference on Decision and Control CDC 1991, Brighton, UK, December 1991.

[11] R. Guidorzi and G. Marro. On wonham stabilizability condition in the synthesis of observers for unknown-input systems. IEEE Trans. on Automatic Control, AC16:499-500, October 1971.

[12] H. Hammouri and Z. Tmar. Unknown input observer for state affine systems: A necessary and sufficient condition. Automatica, 46:271278, 2010.

[13] D. Liberzon and A.S. Morse. Basic problems in stability and design of switched systems. Control Systems Magazine, 19:59-70, 1999.

[14] D. G. Luenberger. Observers for multivariable systems. IEEE Trans. Autom. Control, 11(2):190-197, 1966.

[15] G. Millérioux and J. Daafouz. Flatness of switched linear discrete-time systems. IEEE Trans. on Automatic Control, 54(3):615-619, March 2009.

[16] J. Parriaux and G. Millerioux. Nilpotent semigroups for the characterization of flat outputs of discrete-time switched linear and lpv systems. In Proc. of 51st IEEE Conference on Decision and Control, Grand Wailea, Maui, Hawaii, December 2012.

[17] H. Radjavi and P. Rosenthal. Simultaneous Triangularization. Springer, 2000.

[18] E. Sontag. On the observability of polynomial systems, i: Finite-time problems. SIAM J. Control and Optimization, 17(1):139-151, 1979.

[19] S. Sundaram and C. Hadjicostis. Designing stable inverters and state observers for switched linear systems with unknown inputs. In Proc. of the 45th IEEE Conference on Decision and Control, San Diego, CA, USA, December 2006.

[20] H. Trinh and T. Fernando. Functional Observers for Dynamical Systems, volume 420 of Lecture Notes in Control and Information Sciences. springer, 2012. 